\title{
Enhancing Exercise Experience with Individual Multi-Emotion Provoking Game Elements
}

\author{
Larissa Müller \\ Department Informatik \\ University of Applied Sciences (HAW) \\ Hamburg, Germany \\ School of Engineering and Computing \\ University of the West of Scotland \\ Paisley, Scotland \\ Email: larissa.mueller@haw-hamburg.de
}

Qi Wang

School of Engineering and Computing

University of the West of Scotland

Paisley, Scotland
Arne Bernin

Department Informatik

University of Applied Sciences (HAW)

Hamburg, Germany

School of Engineering and Computing

University of the West of Scotland

Paisley, Scotland

Email: arne.bernin@haw-hamburg.de

\author{
Kai von Luck \\ and Andreas Kamenz \\ and Sobin Ghose \\ Department Informatik \\ University of Applied Sciences (HAW) \\ Hamburg, Germany \\ Email: sobin.ghose@haw-hamburg.de
}

Christos Grecos

Computer Science Department

Central Washington University (CWU)

Ellensburg, USA

\author{
Florian Vogt \\ Innovations Kontakt Stelle (IKS) \\ University of Applied Sciences (HAW) \\ Hamburg, Germany
}

\begin{abstract}
In this work we present a new affective game design method that includes multi-emotion provoking and continuous game elements. Evaluated with our physical cycling exergaming system it extends our previously designed singleemotion game scene approach. In addition we introduce new entertaining content that includes game elements to provoke both physical and mental stress as well as emotions with a controlled intensity to provide an exciting but not taxing exercise. Our previously developed analysis method for facial expressions and physiological data has been enhanced by a near real-time emotion evaluation, to allow the system to adapt the gameplay dynamically and in this work new multi-emotion provoking game elements are introduced. A case study with 25 participants have been conducted to showcase and evaluate the enhancing exercise experience by individual multi-emotion provoking game elements.
\end{abstract}

\section{INTRODUCTION}

The growing business sectors of health care and fitness training shows a trend of increasing expectations for entertaining content. The recent technological surge in self-fitness assessment such as Quantified Self devices providing feedback of activities monitoring and body responses with biosensors. In addition to outdoor training like running or cycling, indoor ergometers are widely in use for endurance, high intensity and warm-up training. Ergometers serve also in recovery training for medical rehabilitation.

By providing entertaining and motivating elements it is possible to keep the endurance training fresh and exiting and thereby promote good training practice [1]. A common motive is to stimulate with thrilling and exciting content, but in healthcare applications it is important not to overdo this stimulation and exceed mental or physical stress provocation, thus causing adverse training effects to patients.

Related research in entertainment computing often applies a scene-based design to provoke emotions, since emotion recognition has been shown to facilitate personalized level design [2]. In this work we present tailored game scenes, which feature game elements as stimuli to trigger specific emotions. In our prior works we established the benefits of a computerized exercise machine to provoke specific emotions in game contexts. Furthermore we presented an analysis method to measure the users' emotional reactions to tailored game scenes by evaluating facial expressions [3] and physiological data [4].

In this work we introduce new entertaining content including game elements with a mixture of physiological stress and emotion provoking game elements both with controlled intensities to provide an exciting but not taxing exercise experience. Our new game design extends our previously designed game scenes with multi-emotion provoking and continuous game designs that make the presented work an important step towards holistic integrated exergames. This work further shows the potential of individualized content in term of enhancing users endurance through an increase of motivation.

\section{RELATED WORK}

The awareness of a person's emotional state allows to adjust the game narrative to ensure a more realistic and satisfactory player experience, as described by Karpouzis and Yannakakis [5]. In this work a nonlinear gameplay concept is presented, which considers individual emotional reactions resulting in different and individual experiences.

It has been shown, that interactive games enhance the experience of general exercises [6] and moreover Lathia et al. [7] linked physical activity to happiness. In our prior works we introduced a physical cycling emotion provoking exergame, the EmotionBike Hamburg [3], [4]. In this work the cycling exergame controller was enhanced by a gear shift 
and a brake to facilitate more thrilling game elements, since the presented virtual game environment is designed as a fun racer. According to Togelius and Yannakakis [2] racing games often adapt their game difficulty based on the performance of the player. Our emotion provoking game was able to adapt to individual emotional responses through facial expressions and physiological data. Thus the designed game was able to alter the game during play and furthermore the presented analysis method allowed game developer to evaluate game elements during the design process.

According to Hong et al. [8] stress has been analyzed as a dimension of negative affect and moreover it has been shown that too much mental strain may increase negative emotions [9]. Providing an exciting but not exhausting exercise experience requires to sense all possible sources of stress the user might perceive, including physical and cognitive strain as well as emotional drain. The influences of physical activity to physiological reactions in stressful situations has for instance been investigated by Hong et al. [8]. In their research the participants were confronted with mental arithmetic problems and noisy sounds as a mental stressors and cold water as a physical stressor. Furthermore stress can be induced for instance by negative feedback in game scenarios and measured by baseline differences in the EDA data [10], but the results are controversial [11]. Results of our previous works [4] have shown that an event-based analysis method of EDA data is an alternative approach and applying an activity peak detection is appropriate for the sensor data focusing on the physic component of the signal. Our experimental design involves the presence of an observer, who is introduced as a judge to provoke a social-evaluative threat. Such a scenario has been shown to induce stress by Dickerson and Kemeny [12].

In summary, a diverse background is required to enable applications with affective features and to promote physical exercise with computer trainers. The overlap of these capabilities is interesting to explore as well on other application contexts. In this work we present special multi-emotion provoking game scenes, which have been tailored to the needs of stress provocation. Moreover our system allows investigating individual emotional reactions for different emotion provoking game events.

\section{EXPERIMENTAL DESIGN}

In the presented work we conducted a case study with 25 participants to showcase the enhancing exercise experience by individual multi-emotion provoking game elements. The individual emotional responses are evaluated by a facial expression analysis and physiological data analysis. Moreover the presented real-time emotion evaluation, allows the system to adapt the gameplay dynamically.

\section{A. Participants Profile}

25 people participated in the conducted case study. These people were ten males and fifteen females aged from 18 to 51 years with an average age of 29 . None of the participants stated to be under medical or drug influences. This is important for the conducted case study, since it influences physiological reactions. Nine of the 25 participants stated to play video games at least two hours per week.

\section{B. Experimental Setup}

Our experimental setup, shown in Figure 1, consists of a cycling exercise machine, a data acquisition system, emotion sensors and an emotion provoking exergame. The cycling exercise machine has been modified to act as a physical cycling exergame controller. The pedal resistance is software controllable and a rotatable handle bar allows steering capabilities. Furthermore a gear shift and a brake has been added to facilitate thrilling game controls and a more natural cycling experience. The data acquisition system logs all game and control events as well as the emotion sensor data. Our emotion sensors consist of a Kinect v2 camera providing video data of frontal images in HD (1080P) resolution and RGB-D $(512 \times 424)$ with a frame-rate up to $30 \mathrm{~Hz}$ and a physiological data acquisition system, the wearable body sensing platform biosignalsplux ${ }^{1}$. The plux system provides electrodermal activity data, operates at a sampling rate of $256 \mathrm{~Hz}$ and transmits the data via Bluetooth to the server. Furthermore the physiological data acquisition system provides a wireless data transmission, which is an important enhancement to the previously applied cable-based approach. The emotion provoking exergame has been enhanced in the presented work and new multi-emotion provoking game scenes will be introduced.

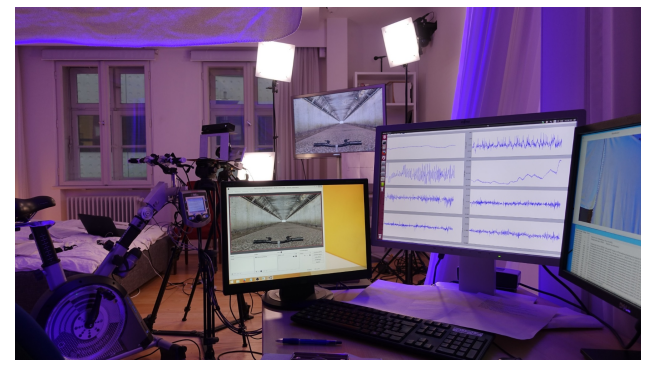

Fig. 1. Experimental Setup: Display Screen, Interactive Cycling Game Controller, Facial Lighting and Control Station for the Experimenter

\section{Emotion Provoking Game}

The game has been designed, to steer participants in controlled emotional states by crafted game scenes. The structure of a linear sequence is changed to a flexible composition of game scenes. A virtual tunnel, shown in Figure 2 is designed and acts as a starting and ending portal to transition between scenes. Every scene starts and ends with a tunnel, which is our chosen place for a brief self-assessment, to ensure a short retrospection time for perceived emotions. The present observer was asked to assess the participants' emotions during the scenes and acts as a judge, since it has been shown by [12], that social-evaluative threats are perceived as stressful. Moreover the difficulty of each scene is configurable in the

\footnotetext{
${ }^{1}$ http://biosignalsplux.com/en/
} 
new game design, but for the presented experiment it was fixed to limit the degrees of freedom.

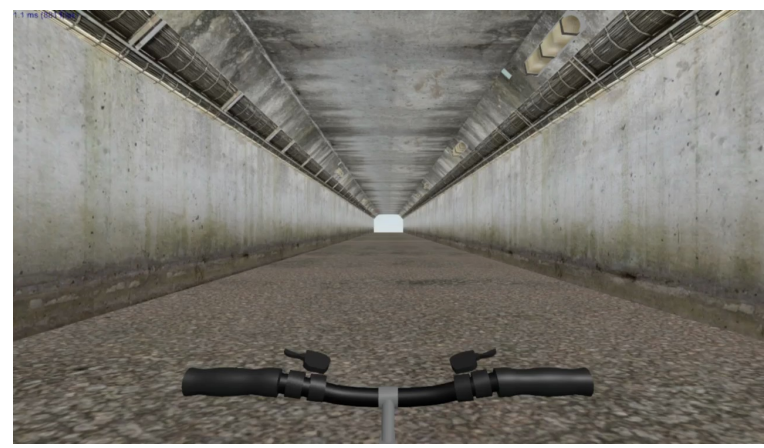

Fig. 2. Tunnel Design: Transition between Scenes

\section{Experimental Tasks}

The experiment consisted of three parts. 1. It started with a dynamic path of controlled emotional provocations, the Emotional Journey, followed by 2. new multi-emotion provoking game scenes. These advanced game scenes were tailored to provide different kinds of emotion provoking elements to support a more natural game experience. The Frozensea Scene includes different types of emotion provoking elements and provides a challenge to the participants. The Cliff Scene is designed to analyze several stressful game elements and also provides a challenging task to the participants in an inconvenient environment.

In the 3. part the participants were divided in two groups. One group had to climb up a mountain, with a high physical pedal resistance and the control group had low pedal resistance, although they climbed the same visual mountain. The emotional and physical conditions of the participants were assessed with questionnaires, including the Borg scale [13] to assess the participants' physical condition. These procedures were repeated after each part of the experiment.

1) Dynamic Path of Emotional Provocations: We designed a concept of an Emotional Journey, in which the game play was adapted to the participants' individual emotional responses in addition to player actions.

The Emotional Journey consists of three mandatory scenes, the established Forest Scene, Challenge Scene and Teddy Scene, which have been shown to provoke specific emotions in [3]. In the Forest Scene a dark forest has to be crossed with a scary Jump Scare Event. The Challenge Scene provides the participants with a very challenging task and the Teddy Scene is full of cute teddy bears. Thus the Emotional Journey aimed to provoke at first surprise or fear, followed by a challenge to provoke ambition, stress or frustration and ends with a joy. During each of these scenes facial expressions and physiological data were analyzed and a decision formed, while the participants were crossing the transition portal tunnel. In case the required emotion could not be detected, another scene tailored for the specific emotion was started. The Explosion Scene was started to provoke surprise or fear by loud explosions in a war zone. The Downhill Scene was designed to provoke stress or frustration by a very challenging task and the Glade Scene to provoke joy by presenting a very nice and relaxing environment. An adaptation to individual emotional reactions has been made to increase the successful provocation rate of the specific emotions and furthermore it shows the ability of the system to adapt to individual emotional responses and behavior.

2) Advanced Emotion Provoking Scenes: Advanced multiemotion provoking game scenes are developed for our game context to provide a natural, exciting and longer lasting game experience.

Our objective was to develop a versatile method to design multi-emotion provocation by postulating three composite strategies:

1. Repetition of the same type of provocation

2. An added obstacle in the pretext of a provocation

3. Providing several emotion provoking game objectives

The details of these strategies are described in the relevant scenes.

The new designed Frozensea Scene provides the participants with different emotion provoking events to compare the intensity in individual emotional reactions. The Coin Collection Event was designed as a challenge task. Four coins have to be collected before a bridge to the finish line is lowered. The coins are placed on an icy ground, which adds another challenge to this task. Another event, which was provided in this scene was a new Jump Scare Event. Snowmen with strange appearance were placed in front of the virtual bicycle accompanied by a loud and shocking sound. Thus in the scene different emotion provoking events can be directly compared.

The Cliff Scene was designed to provoke and analyze stress. Different stressful game elements are presented to the participants in a very challenging surrounding of a narrow mountain path. A very sensitive steering is required to reach the finish line and in most cases it takes many attempts to fulfill the task without falling down the cliff.

In both the Cliff Scene and the Frozensea Scene occurs the Falling Event and thus the 1. composite strategy of repetition of the same type of provocation is applied in this part of the experiment.

\section{Frozensea Scene:}

In the Frozensea Scene the virtual bicycle is placed in a cold environment full of snow and ice, shown in Figure 3. The objective of the scene is to collect four coins, which are placed on icy ground as shown in Figure 10. The controls of the virtual bicycle show a strange behavior on that ground, simulating a real bike on ice. It is very difficult to align the proper direction to a coin, due to very sensitive steering. Moreover the brake is disabled. The objective of the scene is to collect all coins, based on the results of our previous works [3]. The icy ground separates a canyon from the finish line. After the collection of all coins a bridge is lowered to overcome the canyon. In case the player falls down the bridge or the cliff edge the Falling Event is triggered and the scene starts again. During the ride through the icy world a surprising Snowmen 
Event is triggered. As presented in Figure 4, strange looking snowmen appear in front of the virtual bike accompanied by a horrible loud sound. The various events inside the scene have been designed to evaluate, which one provokes the strongest reactions.

Applying the 2. composite strategy of adding an obstacle in the pretext of a provocation, the Falling Event occurs unexpectedly, since the participants think they have completed the task and did not anticipate another hidden challenge.

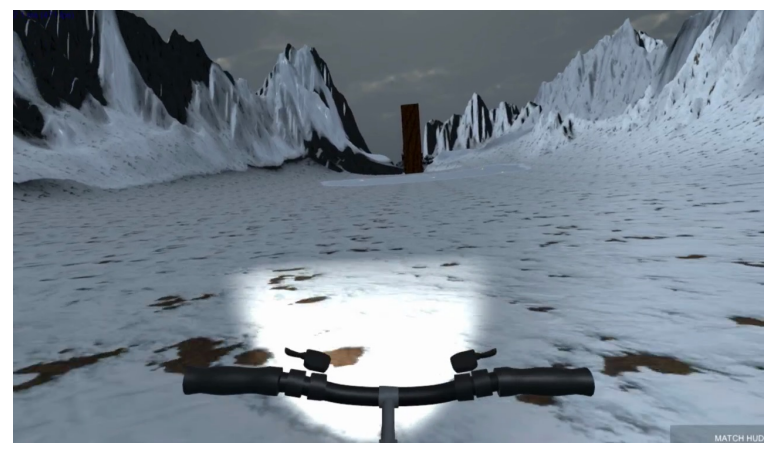

Fig. 3. Frozensea Scene

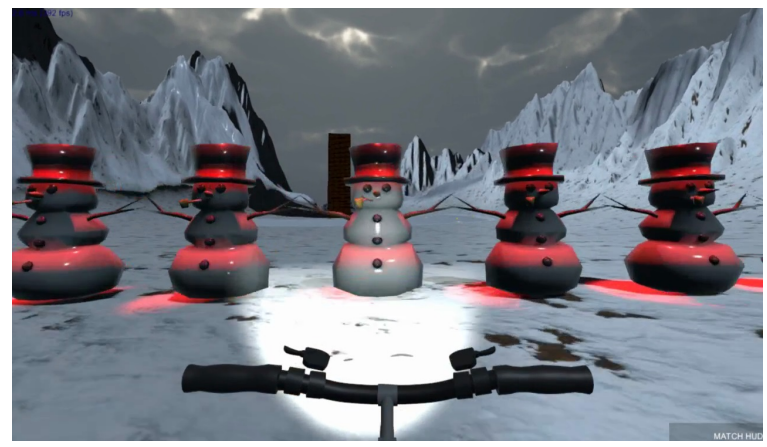

Fig. 4. Surprisingly Snowmen Event in the Frozensea Scene

\section{Cliff Scene:}

The Cliff Scene starts high up an icy mountain, as presented in Figure 5. A narrow pathway with a steep cliff edge is the only way to the finish line. On top of the mountain, loosen rocks falls off and sometimes hit the bike to increase the participants' stress level. At the beginning a Demo Rock Event is triggered to showcase the possibility of getting hit by a rock. The falling rocks are software controllable and are triggered by the experimenter to maximize the stressful experience. In case a rock hits the virtual bike the Rock Hit Player Event was logged into the system and the player mostly falls downwards the cliff edge accompanied by the Falling Event, which starts the scene again.

The participants need to drive very slowly and need to be very focused on the steering to reach the finish line, since the pathway narrows during the ride and sharp curves complicate the task. Furthermore at the end of the scene, when the players can see the finish line, they have to drive over a very narrow bridge as shown in Figure 11. This provokes fear and the system logs the Bridge Entered Event. Since reaching the finish line is a very challenging task, a teleporting option was offered to the participants after an exceeding amount of trials to end the scene directly.

Several stressful game elements challenge the participants in the Cliff Scene, thereby applying the 3. composite strategy of providing several emotion provoking game objectives (Rock Hit Player Event, Bridge Entered Event, Falling Event, Demo Rock Event).

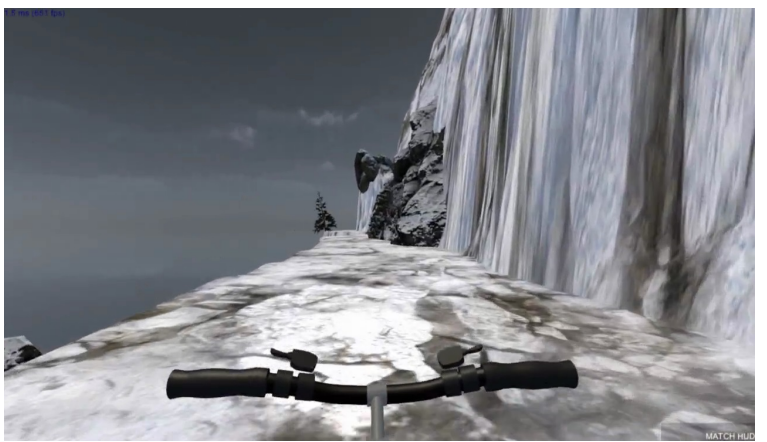

Fig. 5. Icy Mountain of the Cliff Scene

3) Physical Stress and Emotional Provocation: For the last part of the experiment the participants were separated in two groups. The objective was the same, two courses had to be completed: the Treehouse Scene and the Mountain Scene. The physical resistance of the pedals of one group was set to a low maximum, although they had to climb up a mountain. For the other group the resistance was set related to the slope of the virtual trail. In case the participants had a high resistance the experimenter informed them to choose a high gear and startup the hill with a high speed. The aim of this design was to evaluate assumed influences of physical effort to surprising events.

\section{Treehouse Scene:}

In the Treehouse Scene the player has to follow a path to the finish line. The ascent of the path is configurable. In the presented work the ascent was set to a high value. For one group, the physical pedal resistance was increased to a defined maximum of 150 Watt and for the control group it was set to a lower maximum of 100 Watt. In case of a high maximum the player had to increase the gearshift and to drive very fast, otherwise the virtual bicycle does not manage to climb up the ascent and make it to the finish line.

\section{Mountain Scene:}

The Mountain Scene, starts on a mountain road and the participants have to climb up a hill with the bike. The physical resistance of the ergometer pedals increases as a percentage of the degree of ascent in order to analyze the influence of physical strain for one group, similar to the Treehouse Scene. After the participants reached a plateau, which is filled with tall and dense grass, spiders attack the bike. The Spider Attack's, shown in Figure 7, are triggered to surprise the player, since a configurable amount of spiders are hard to spot in order to detour, due to the occluded environment. 


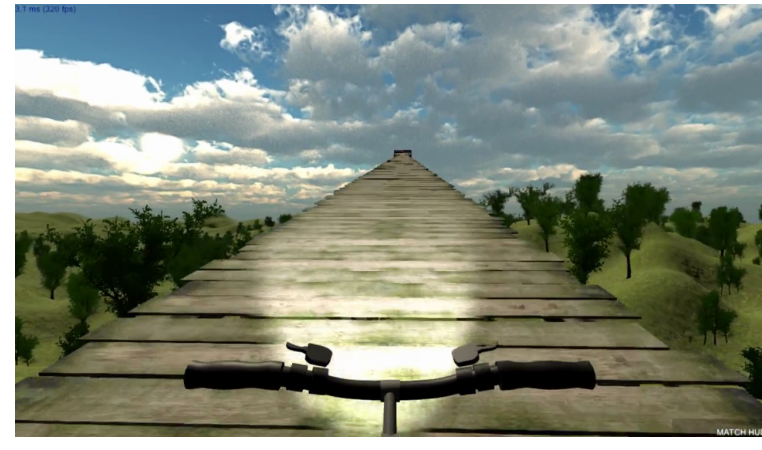

Fig. 6. Treehouse Scene

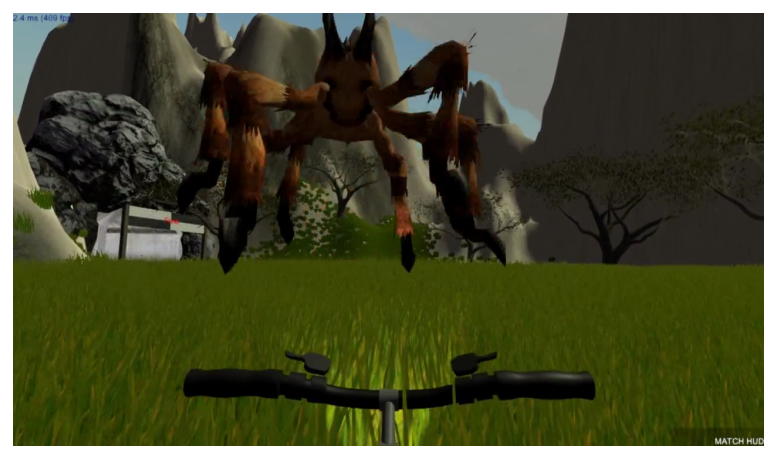

Fig. 7. Spider Attack Event in the Mountain Scene

\section{DATA ANALYSis}

In our previous work [3] we developed an facial expressions based analysis method for emotion recognition, which provides an event-based evaluation with an analysis window of 0.5 seconds before and 3.5 seconds after an event occurred. Furthermore we enhanced the facial expression analysis method by near-real time abilities, since we integrated the emotion recognition software Emotient from iMotions ${ }^{2}$ to our system, based on the findings presented in [14]. Emotient provides the emotions: anger, joy, surprise, sadness, disgust, fear, contempt, confusion, frustration and neutral.

A method for emotion detection by physiological data has also been introduced in a previous work [4]. Due to the previously presented results, this work is focused on the analysis of EDA data with an analysis window from one second before and nine seconds after an event occurred. Furthermore the method has been enhanced to support a near real-time analysis to facilitate an adaptive system response to emotional reactions.

In the facial expression analysis, a scene specific clustering has been applied in case a tailored game scene was aimed to provoke different characteristics of an emotion. This is an enhancement to the application-based clustering method described in [14]. Moreover the event-based facial expression analysis has been combined with the event-based analysis of EDA data.

\footnotetext{
${ }^{2} \mathrm{https} / / /$ imotions.com/emotient/
}

\section{A. Analysis Results}

The facial expression and physiological data analysis showed promising results in the introduced multi-emotion provoking game scenes. The results provide an auspiciously combination of physical stress and emotional provocation. Furthermore the Emotional Journey concept has shown exciting results, as described in the following:

1) Dynamic Path of Emotional Provocations: A detailed interview followed subsequent to the Emotional Journey part of the experiment. It consisted of several questionnaires regarding the game concept, their experience and comprehensive selfassessment. This qualitative analysis aimed at the evaluation of the presented concept of an Emotional Journey. The participants were asked about their ability to recognize and reproduce the in-scene provocation. The overall recognition rate of the designed Emotional Journey was 89,3\%. All of the participants did recognize the aim of the Jump Scare Event, to provoke fear, surprise or scare, resulting in a recognition rate of $100 \%$. The Challenge Scene was likewise very successful with a recognition rate of $100 \%$, since all participants stated that frustration or ambition should be provoked by these scenes. The aim of the Teddy Scene and the Glade Scene was to provoke joy, which was recognized by $68 \%$ of the participants.

2) Multi-emotion Provoking Game Scenes: The two multiemotion provoking game scenes have been stated as favorite scenes by most of the participants. The Cliff Scene had the highest ranking with seven votes and the Frozensea Scene was ranked at the second place with five votes. Figure 8 illustrates two events and its physiological reactions in EDA raw data of the Cliff Scene, the Rock Hit Player Event and the Bridge Entered Event. Furthermore Figure 8 displays that different intensities of emotion provocation could be found in the tailored game scenes, which can be directly compared. The analysis results show, that the Rock Hit Player Event has been perceived as the most stressful in the Cliff Scene. Neither the Falling Event nor the challenging bridge, which had to be crossed, provoked such intense reactions. In the Frozensea Scene the surprisingly occurring Snowmen Event and the Coin Collection could not trigger the same reliable results as the Falling Event. The different intensities of emotional reactions to similar events in different game scenes, shows the importance of considering the whole game context and all emotions in their order of provocation. Thus it is important to analyze multi-emotion provocation in affective games.

Table I illustrates the results of the scene specific clustering of the facial expression analysis. Table II presents combined results of the facial expression analysis and the EDA data analysis. In the following detailed results of the Frozensea Scene and the Cliff Scene are illustrated.

The repetition of events has been shown as affectively in our previous works, in this work postulated as composite strategy 1. The results of this case study indicates that the 2 . composite strategy leads to more intense reactions. In particular the unexpected Falling Event show strong responses (100\%) in the Frozensea Scene, in contrast to the expected Falling Event, which showed less strong responses $(90,9 \%)$ in the Cliff Scene. 


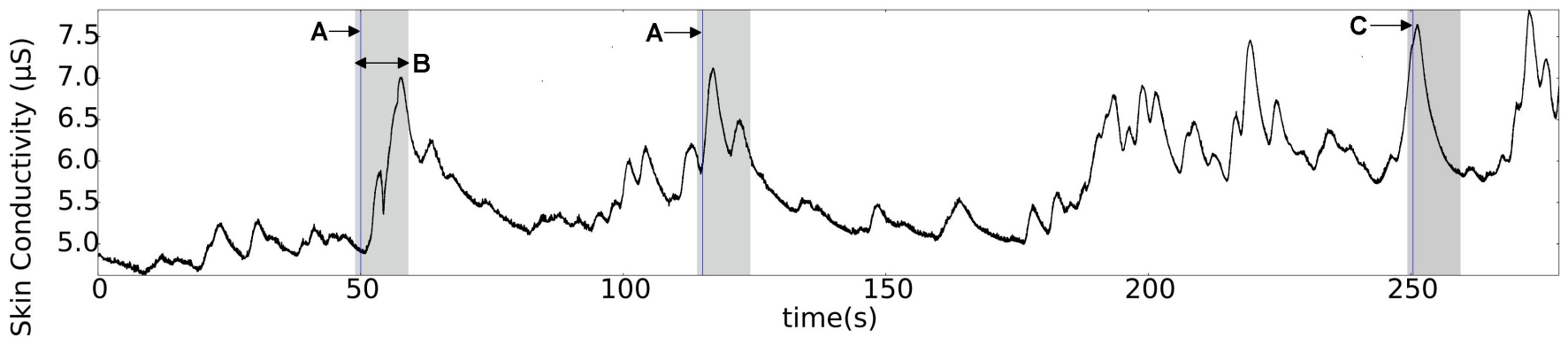

Fig. 8. Typical time series of the Cliff Scene with multiple events consists of the EDA data, A: Rock Hit Player Events, B: Analysis Window, C: Bridge Entered Event

TABLE I

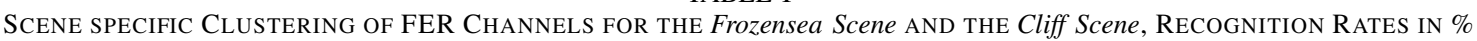

\begin{tabular}{|c|c|c|c|c|c|c|}
\hline Scene & Event & $\begin{array}{l}\text { FER 1 } \\
\text { Emotion }\end{array}$ & $\begin{array}{l}\text { FER 2 } \\
\text { Emotion }\end{array}$ & FER 1 & FER 2 & $\begin{array}{l}\text { Combined } \\
\text { FER 1 / FER 2 }\end{array}$ \\
\hline Frozensea & Snowmen & Fear & Surprise & 39,5 & 17,1 & 44,7 \\
\hline Frozensea & Coin Collection & Joy & & 43,3 & & 43,3 \\
\hline Frozensea & Falling & Frustration & Joy & 25,0 & 87,5 & 93,8 \\
\hline Cliff & Falling & Frustration & Joy & 25,0 & 65,9 & 70,5 \\
\hline Cliff & Bridge Entered & Fear & Surprise & 36,4 & 13,6 & 45,5 \\
\hline Cliff & Rock Hit Player & Fear & Surprise & 56,3 & 18,8 & 56,3 \\
\hline Cliff & Demo Rock & Fear & Surprise & 37,5 & 12,5 & 41,7 \\
\hline Mountain & Spider Attack & Fear & Surprise & 50,0 & 33,3 & 54,2 \\
\hline
\end{tabular}

TABLE II

Two Modalities COMBined: FER AND EDA FOR THE Frozensea Scene AND THE Cliff Scene, RECOGNITION RATES IN \%

\begin{tabular}{|c|c|c|c|c|}
\hline Scene & Event Name & FER & EDA & Combined FER/EDA \\
\hline Frozensea & Snowmen & 44,7 & 84,2 & 90,8 \\
\hline Frozensea & Coin Collection & 43,3 & 62,2 & 72,0 \\
\hline Frozensea & Falling & 93,8 & 87,5 & 100 \\
\hline Cliff & Falling & 70,5 & 79,5 & 90,9 \\
\hline Cliff & Bridge Entered & 45,5 & 77,3 & 90,9 \\
\hline Cliff & Rock Hit Player & 56,3 & 87,5 & 93,8 \\
\hline Cliff & Demo Rock & 41,7 & 62,5 & 75 \\
\hline Mountain & Spider Attack & 54,2 & 83,3 & 87,5 \\
\hline
\end{tabular}

Frozensea Scene: The detection of only fear Snowmen Event in the Frozensea Scene shows a rate of 39,5\% and the detection of only surprise $17,1 \%$. This event is software controllable and was triggered by the experimenter to maximize the surprising effect and the number of analyzed events was 76. The detection of both fear and surprise improved the results to $44,7 \%$, since both emotions are acceptable in this case. The results of this scene specific clustering is presented in Table I for all scenes. The analysis of EDA data showed peaks in $84,2 \%$ and sample raw data are presented in Figure 9. The combination of EDA and FER analysis increased the rate to $90,8 \%$, as shown in Table II.

The Coin Collection Event provoked joy in $43,3 \%$ of the events. The number of events is very high $(\mathrm{n}=164)$, due to the fact that the scene starts again in case the participants fall down the cliff. Eleven participants made it at the first trial. Ten needed two attempts and one needed three. For one person the task was very challenging, since it took four attempts to reach the finish line. Peaks in EDA data was found in $62,2 \%$ of the Coin Collection Event's. The combination of EDA and FER analysis increased the number to $72,0 \%$.

The Falling Event provoked frustration in $25 \%$ and joy in $87,5 \%$ of the 16 events $(n=16)$. The number of events is lower, since not all participants fell down the cliff. The scene specific clustering of frustration and joy, which has been further described in our previous works results in $93,8 \%$. The combination of EDA and FER analysis increased the detection rate to $100 \%$. Although the occurrence of the Falling Event is much less than in other scenes, it was perceived as very frustrating. It takes considerable time to finish the collection of all the coins (2. composite strategy), which might lead to a much stronger perception of frustration even with minor occurrences of the Falling Event compared to other scenes.

Cliff Scene: Frustration was displayed in facial expressions in $25,0 \%$ and joy in $65,9 \%$ of the Falling Events $(\mathrm{n}=44)$. The rate increased by the scene specific clustering to $70,5 \%$. The EDA showed peaks in $79,5 \%$ of the events. A combination of FER and EDA analysis results in a detection rate of $90,9 \%$.

The Bridge Entered Event provoked fear in $36,4 \%$ and surprise in $13,6 \%(\mathrm{n}=22)$ and a detection of both results in $45,5 \%$. Peaks were displayed in $77,3 \%$ of the EDA data. The combination of FER and EDA increased the rate to $90,9 \%$.

Fear was displayed in 56,3\% and surprise in $18,8 \%$ of the Rock Hit Player Event $(\mathrm{n}=16)$. The scene specific clustering results in 56,3\%. The EDA data showed peaks in $87,5 \%$ of the events. The combination of FER and EDA analysis results in $93,8 \%$ detection rate.

The Demo Rock Triggered Event provoked fear 37,5\% 


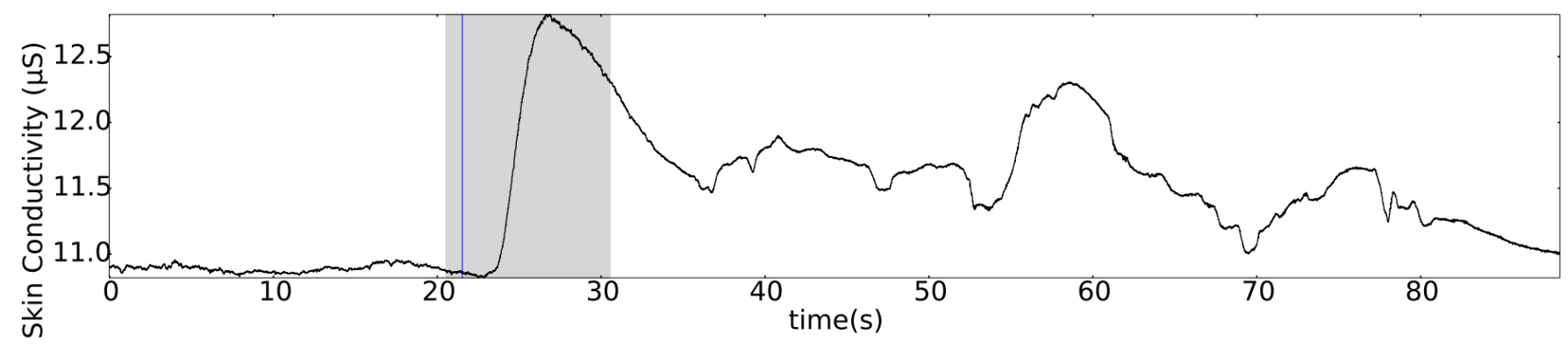

Fig. 9. Typical time series of the Frozensea Scene for the Snowmen Event

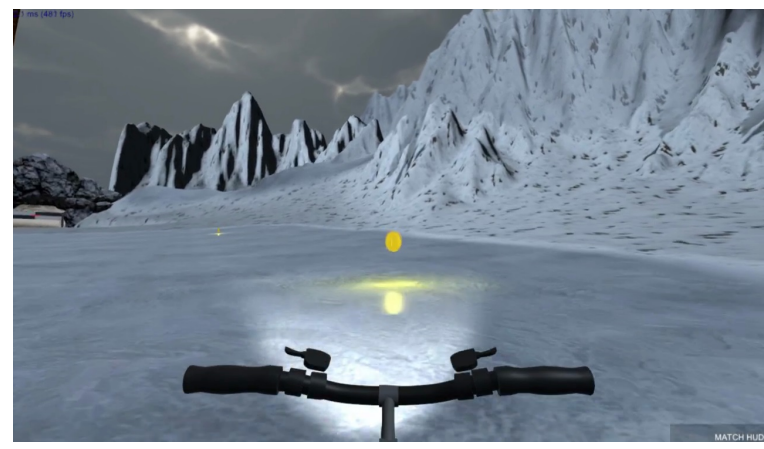

Fig. 10. Coin Collection Event in the Frozensea Scene

and surprise in $12,5 \%$ of the events $(n=24)$. The combined detection results in $41,7 \%$. The EDA analysis detected peaks in $62,5 \%$ of the events. The combination of FER and EDA analysis increased the detection rate to $75 \%$.

The 3. composite strategy of providing several emotion provoking game objectives showed that the Rock Hit Player Event provoked the most intense reactions compared to the events Falling Event,Bridge Entered Event and Demo Rock Triggered Event. The details of these strategies are described in the relevant scenes.

Only two of the participants agreed to the option to be teleported to the finish line. Although many attempts were necessary the rest wanted to make it on their own, which emphasizes, the exciting and compelling effect of the entertaining content.

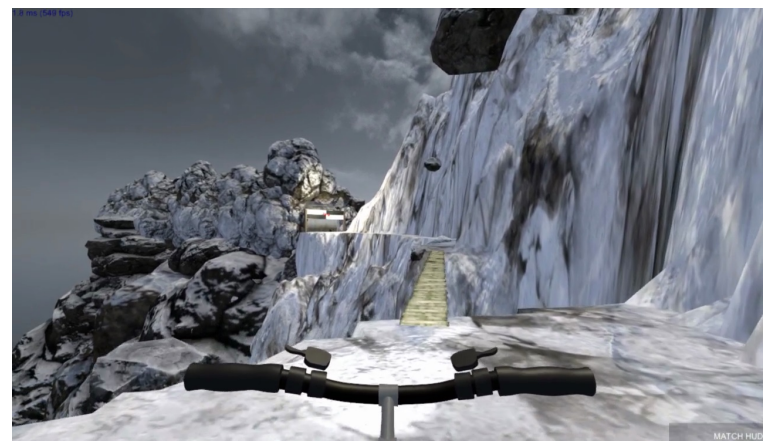

Fig. 11. Scary Bridge in front of the Finish Line
3) Physical Stress and Emotional Provocation: A detection rate of $87,5 \%$ for the Spider Attack Event followed the combination of EDA and facial expressions, as shown in Table II. This high detection rate shows at a qualitative level, that the physical effort of the Treehouse Scene and the Mountain Scene does not have a huge influence on the emotional reactions. A more quantified assertion requires an experimental design with more participants.

Many participants from the control group stated they perceived a challenge or physical strain to climb the mountain although the pedal resistance stayed low. This is an interesting finding. In the following the detailed results of the Treehouse Scene and the Mountain Scene are presented.

Treehouse Scene: The aim of the Treehouse Scene was to stimulate physical effort on the exercise machine, by presenting an extreme ascent in the virtual environment. Since no emotion provoking game elements were included in the scene, none have been evaluated.

Mountain Scene: The Spider Attack Event in the Mountain Scene provoked fear in $50 \%$ of the FER data. Surprise was found in $33,3 \%$. The scene specific clustering of these two emotions let to a detection rate of $54,2 \%$. The EDA data showed peaks in $83,3 \%$ of the events $(n=24)$. A combination of FER and EDA data increased the detection to $87,5 \%$. Figure 12 shows a typical time series of EDA raw data and facial expression probability of fear in the Mountain Scene for the Spider Attack Event and illustrates the beneficial effect of combining both analysis output. The facial expression output shows a peak of fear probability for both events, while the EDA displays a peak only at the second event.

\section{CONClusion}

In this work we introduced new entertaining content for fitness applications by multi-emotion provoking game scenes, which showed promising results in the applied facial expression and physiological data analysis. In particular we combined physical stress with emotional provocation to create an exciting but not an too exhausting or stressful training.

The presented near real time analysis method for facial expressions and EDA measurements improved the emotion recognition accuracy for established single-emotion and new multi-emotion provoking game scenes. Our new approach of a scene specific clustering of emotional reactions in facial 

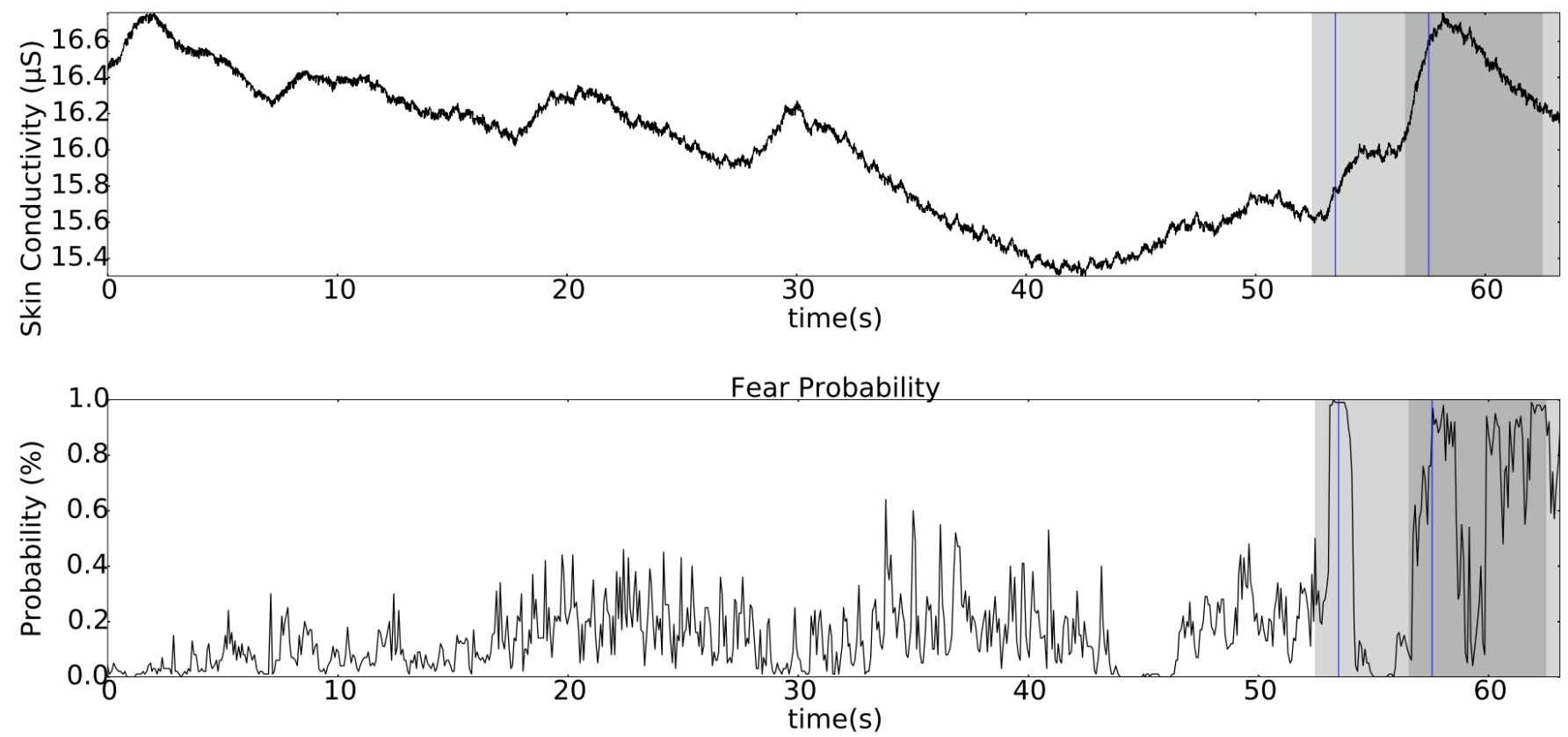

Fig. 12. Typical time series of the Mountain Scene for the Spider Attack Event, consists of a. the EDA raw data and b. the facial expression probability of fear

expressions and its combination with EDA data analysis generates significantly more robust emotion recognition rates.

The results of the conducted case study have shown that individual differences in emotional responses and intensities in reactions to distinct game elements can be reliably detected with our system.

We introduced three new composite strategies to effectively integrate stress and emotion provocations in game scenes, which is a useful method to tailor new entertaining content for fitness applications - "the dose matters" to cause the wanted effect. An example is the Rock Hit Player Event, which has been perceived as the most stressful in the Cliff Scene. In contrast, the Falling Event did not provoke that intense reactions, although a similar event has shown powerful responses in other scenes. This emphasizes the importance of evaluating multi-emotion provocation game elements in affective games. In summary, with this work we have shown that provocation and physical stress can be mixed in game scenes with some design care in timing and story telling elements based on individual reactions. Our work is a step towards more engaging and emotion responsive fitness applications.

\section{REFERENCES}

[1] R. Malaka, "How computer games can improve your health and fitness," in International Conference on Serious Games. Springer, 2014, pp. 1-7.

[2] J. Togelius and G. N. Yannakakis, "Emotion-driven level generation," in Emotion in Games. Springer, 2016, pp. 155-166.

[3] L. Müller, S. Zagaria, A. Bernin, A. Amira, N. Ramzan, C. Grecos, and F. Vogt, "Emotionbike: a study of provoking emotions in cycling exergames," in Entertainment Computing-ICEC 2015. Springer, 2015, pp. $155-168$.
[4] L. Müller, A. Bernin, S. Ghose, W. Gozdzielewski, Q. Wang, C. Grecos, $\mathrm{K}$. von Luck, and F. Vogt, "Physiological data analysis for an emotional provoking exergame," in Computational Intelligence (SSCI), 2016 IEEE Symposium Series on. IEEE, 2016, pp. 1-8.

[5] I. Kotsia, S. Zafeiriou, G. Goudelis, I. Patras, and K. Karpouzis, "Multimodal sensing in affective gaming," in Emotion in Games. Springer, 2016, pp. 59-84.

[6] D. E. Warburton, S. S. Bredin, L. T. Horita, D. Zbogar, J. M. Scott, B. T. Esch, and R. E. Rhodes, "The health benefits of interactive video game exercise," Applied Physiology, Nutrition, and Metabolism, vol. 32, no. 4, pp. 655-663, 2007.

[7] N. Lathia, G. M. Sandstrom, C. Mascolo, and P. J. Rentfrow, "Happier people live more active lives: Using smartphones to link happiness and physical activity," 2016.

[8] J.-H. Hong, J. Ramos, and A. K. Dey, "Understanding physiological responses to stressors during physical activity," in Proceedings of the 2012 ACM conference on ubiquitous computing. ACM, 2012, pp. 270279.

[9] P. J. Feldman, S. Cohen, S. J. Lepore, K. A. Matthews, T. W. Kamarck, and A. L. Marsland, "Negative emotions and acute physiological responses to stress," Annals of Behavioral Medicine, vol. 21, no. 3, pp. 216-222, 1999.

[10] H. G. Wallbott and K. R. Scherer, "Stress specificities: Differential effects of coping style, gender, and type of stressor on autonomic arousal, facial expression, and subjective feeling." Journal of Personality and Social Psychology, vol. 61, no. 1, p. 147, 1991.

[11] T. Friedrichs, C. Zschippig, M. Herrlich, B. Walther-Franks, R. Malaka, and K. Schill, "Simple games-complex emotions: Automated affect detection using physiological signals," in International Conference on Entertainment Computing. Springer, 2015, pp. 375-382.

[12] S. S. Dickerson and M. E. Kemeny, "Acute stressors and cortisol responses: a theoretical integration and synthesis of laboratory research." Psychological bulletin, vol. 130, no. 3, p. 355, 2004.

[13] G. Borg, "Anstrengungsempfinden und körperliche aktivität," Deutsches Ärzteblatt, vol. 101, no. 15, pp. 1016-21, 2004.

[14] A. Bernin, L. Müller, , S. Ghose, K. von Luck, Q. Wang, C. Grecos, and F. Vogt, "Towards more robust automatic facial expression recognition in smart environments," in Proceeding of the PErvasive Technologies Related to Assistive Environments (PETRA) conference, Rhodos. ACM, 2017. 